\title{
Response of an annular electrostatic probe for a planar dielectric spacer: II
}

\author{
Johansson, Torben; McAllister, lain Wilson
}

Published in:

Annual Report Conference on Electrical Insulation and Dielectric Phenomena, 2004. CEIDP '04.

Link to article, DOI:

10.1109/CEIDP.2004.1364219

Publication date:

2004

Document Version

Publisher's PDF, also known as Version of record

Link back to DTU Orbit

Citation (APA):

Johansson, T., \& McAllister, I. W. (2004). Response of an annular electrostatic probe for a planar dielectric spacer: II. In Annual Report Conference on Electrical Insulation and Dielectric Phenomena, 2004. CEIDP '04. IEEE. https://doi.org/10.1109/CEIDP.2004.1364219

\section{General rights}

Copyright and moral rights for the publications made accessible in the public portal are retained by the authors and/or other copyright owners and it is a condition of accessing publications that users recognise and abide by the legal requirements associated with these rights.

- Users may download and print one copy of any publication from the public portal for the purpose of private study or research.

- You may not further distribute the material or use it for any profit-making activity or commercial gain

- You may freely distribute the URL identifying the publication in the public portal 


\title{
2004 Annual Report Conference on Electrical Insulation and Dielectric Phenomena \\ Response of an annular electrostatic probe for a planar dielectric spacer: II
}

\author{
T. Johansson and I. W. McAllister \\ Ørsted•DTU \\ Technical University of Denmark, Lyngby, Denmark
}

\begin{abstract}
The response of annular electrostatic probes mounted in an electrode is examined with reference to a planar dielectric spacer. On this occasion the presence of a high-voltage point electrode is included in the system geometry. The study involves using the probe $\lambda$ function to derive characteristic parameters. These parameters enable the response of such probes to be quantitatively assessed.
\end{abstract}

\section{Introduction}

Recently, the response of bound annular electrostatic probes has been examined with respect to a plane dielectric spacer alone [1]. Such probes were used by Yamamoto and colleagues to examine the surface charge resulting from the development of a discharge across the surface of a planar gas/solid-dielectric interface [2-4]. In these studies a high-voltage point electrode was employed to promote discharge development across the surface of the solid dielectric.

The word bound signifies that the probe is mounted in the electrode in contact with the solid dielectric. Thus, as the probe is an integral part of this electrode, such probes lack the freedom of movement necessary to implement a scanning procedure. This is required for a correct quantitative evaluation of the surface charge to be made [5-7].

The relationship between the charge induced on a probe and the charge density at the gas/solid-dielecric interface is expressible in terms of a general function, the $\lambda$ function [8]. Using this function it is possible to examine the response of electrostatic probes. In the present study, the response of annular probes to surface charge on a planar dielectric spacer, in the presence of a high-voltage electrode, is investigated. For a system of concentric annular probes, the point electrode is shown to have a strong effect on the $\lambda$ functions of the inner electrodes. In turn this influences the charge induced on these probes, and hence the response is considerably modified by the presence of the high-voltage electrode.

\section{The $\lambda$ function}

The $\lambda$ function is the proportionality factor between the charge induced on the probe to any charge within the dielectric volume or at an interface [8]. If it is assumed that the volume charge density in the solid dielectric is zero, then this relationship can be expressed simply as

$$
q=-\iint_{A_{\mathrm{o}}} \lambda \sigma \mathrm{d} A
$$

where $q$ is the Poissonian induced charge on the probe sensor plate, and $\sigma$ is the surface charge density on the surface element $d A$ of $A_{0}$, the surface of the solid dielectric.

The dimensionless parameter $\lambda$ is a solution of the general Laplace equation for the complete system geometry:

$$
\vec{\nabla} \bullet(\varepsilon \vec{\nabla} \lambda)=0
$$

The boundary conditions are $\lambda=1$ at the sensor plate of the probe in question and $\lambda=0$ at all other electrodes. In addition, at dielectric interfaces the normal derivatives of $\lambda$ must obey the condition

$$
\varepsilon_{+}\left(\frac{\partial \lambda}{\partial n}\right)_{+}=\varepsilon_{-}\left(\frac{\partial \lambda}{\partial n}\right)_{-}
$$

where the + and - signs refer to the opposite sides of the interface. As (2) is just Laplace's equation, any standard method of solving this equation can be employed to evaluate the variation of $\lambda$ along the interface. On this occasion, solutions of Laplace's equation were obtained using a finite element software package. These solutions were then used to study the dependence of the probe response upon the surface charge distribution.

\section{Probe/dielectric geometry}

To establish the characteristics of a system of annular probes with an in situ high-voltage electrode, we will consider a system involving 8 concentric probes. The essential geometry of the system is shown schematically in Figure 1, in which $r_{\mathrm{i}}$ and $r_{0}$ are the inner and outer radii of an annular probe sensor plate, 
while $w$ represents the width of this sensor, i.e. $w=r_{0}-r_{\mathrm{i}}$. In the present study, $w=2 \mathrm{~mm}$. The gap between the sensor plate and a neighbouring probe is $\Delta$. As in [2-4] this spacing is fixed at $0.2 \mathrm{~mm}$. If $i$ represents the probe number, with $i=1,2,3, \ldots 8$, then the relative geometric dimensions of the 8 probes are listed in Table 1. The central probe ( $i=1)$ with $r_{\mathrm{i}}=0$ is just a circular disc. It should be noted that the probe dimensions are identical to those used in [1].

The high-voltage point electrode is a hemispherically-ended cylindrical rod, of radius $R=5 \mathrm{~mm}$, in contact with the upper surface of the dielectric plate, see Figure 1. The length of this electrode is much longer than the thickness of the dielectric plate.

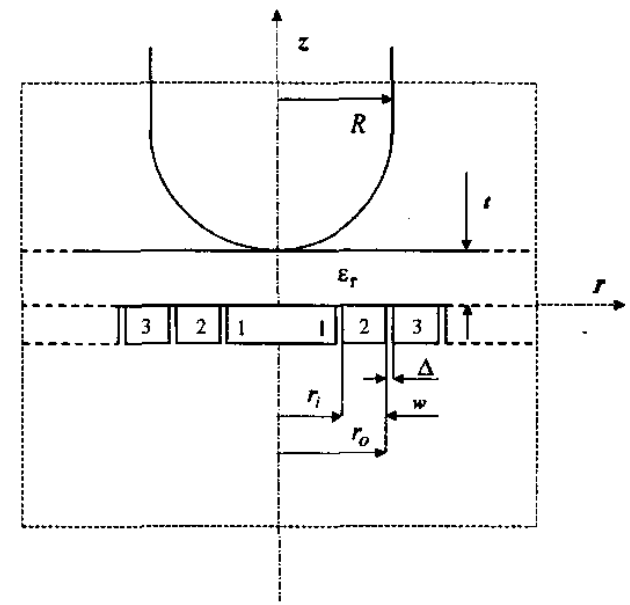

Figure 1: Annular-probes/planar-dielectric geometry.

The planar dielectric, of relative permittivity $\varepsilon_{\mathrm{r}}$, has a thickness $t$ in a direction normal to the plane electrode which is grounded. As in [2-4], $t=2 \mathrm{~mm}$. In the direction normal to probe/gap axis, the extent of

Table 1: Relative geometry of annular probes.

$\begin{array}{lll}i & r_{\mathrm{i}} / w & r_{0} / w \\ 1 & 0 & 1.0 \\ 2 & 1.1 & 2.1 \\ 3 & 2.2 & 3.2 \\ 4 & 3.3 & 4.3 \\ 5 & 4.4 & 5.4 \\ 6 & 5.5 & 6.5 \\ 7 & 6.6 & 7.6 \\ 8 & 7.7 & 8.7\end{array}$

$r_{\mathrm{i}}$ - inner probe radius

$r_{0}$ - outer probe radius

probe width $w=2 \mathrm{~mm}$

probe separation $\Delta=0.2 \mathrm{~mm}$

point radius $R=5 \mathrm{~mm}$

thickness of dielectric $t=2 \mathrm{~mm}$ the plane electrode/planar dielectric is much greater than the probe lateral dimensions.

\section{Results}

The variation of the $\lambda_{i}$ along the planar dielectric interface, where $i$ denotes the probe number, for $\varepsilon_{\mathrm{r}}=$ 4 is shown in Figure 2 . As the charge induced by the same source differs from probe to probe, it is necessary to evaluate the $\lambda$ function for each probe: i.e. we have $\lambda_{1}, \lambda_{2}, \ldots \lambda_{8}$. Figure 2 refers to the four inner probes $(i=1, \ldots 4)$ as the $\lambda$ functions exhibit clearly the influence of the point electrode $(\lambda=0)$.

A comparison with the corresponding Figures in [1] indicates that the existence of $\lambda=0$ at $r=0, z=t$ suppresses the maximum $\lambda$ values associated with the inner probes and as a consequence the central probe no longer exhibits a bell-shaped $\lambda$ distribution.

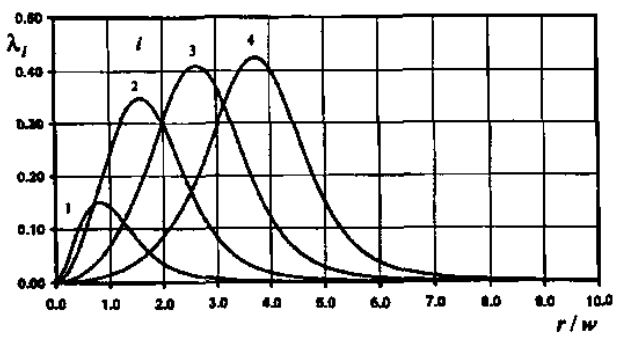

Figure 2: Variation of $\lambda_{i}$ along the planar interface for the inner probes.

For the four outer probes $(i=5, \ldots 8)$, a comparison with the $\lambda$ functions shown in [1] indicates that the influence of the point electrode is negligible: i.e. the $\lambda$ functions for these probes are effectively identical with those presented in [1].

The behaviour of $\lambda_{i}$ with permittivity is as expected for bound probes: i.e. an increase in $\varepsilon_{\mathrm{r}}$ produces an increase in the magnitude of $\lambda_{i}$.

Charge is also induced on the point electrode, and therefore the relevant $\lambda$ function, $\lambda_{p}$, has been evaluated. In contrast to the $\lambda_{i}$ distributions, the variation of $\lambda_{p}$ along the dielectric interface is quite different, with a maximum value of unity on the axis, see Figure 3. This Figure also demonstrates that, unlike the annular probes, an increase in $\varepsilon_{\mathrm{r}}$ now leads to a reduction in the $\lambda_{p}$ values away from the axis.

The significance of the variations in the $\lambda_{i}$ functions can be quantified by examining the behaviour of another parameter which describes the 


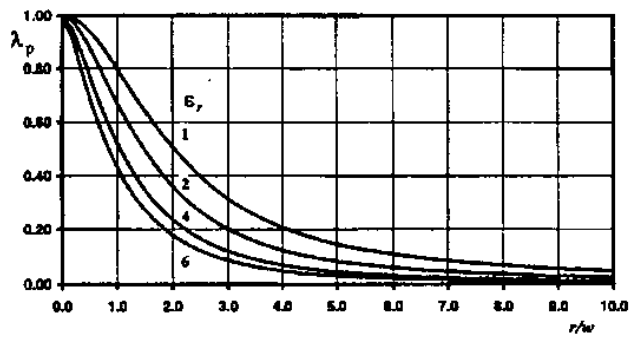

Figure 3: Variation of $\lambda_{p}$ along the planar interface

for the point electrode

detection sensitivity $S_{\mathrm{e}}$ of the probe with respect to a surface charge at the dielectric interface.

If $(r, z)$ represent cylindrical coordinates, see Figure 1 , then for a circular disc of constant surface charge density $\sigma_{0}$, the charge $q_{i}$ induced on probe $i$ is given by

$$
q_{i}(r)=-2 \pi \sigma_{\mathrm{o}} \int_{0}^{r} \lambda_{i} r^{\prime} \mathrm{d} r^{\prime}
$$

where $r^{\prime}$ is a dummy variable.

Traditionally, electrostatic probe measurements are interpreted as though the probe responds only to the charge directly subtended by the sensor plate, i.e. the value of charge $Q_{i}$ assumed to be measured is given by

$$
Q_{i}=\pi\left(r_{\mathrm{o}}^{2}-r_{\mathrm{i}}^{2}\right)_{i} \sigma_{\mathrm{o}}
$$

By using this charge value as a normalising factor, it is possible, with respect to induced charge, to define a detection sensitivity as

$$
S_{\mathrm{e} i}=\frac{\left|q_{i}\right|}{Q_{i}}=\frac{2}{\left(r_{\mathrm{o}}^{2}-r_{\mathrm{i}}^{2}\right)_{i}} \int_{\mathrm{o}}^{r} \lambda_{i} r^{\prime} \mathrm{d} r^{\prime}
$$

A typical radial extent of the streamer discharge reported in [2-4] was approximately $15 \mathrm{~mm}$ : i.e. if $r_{0}$ denotes the radial extent of the surface charge, then we assume $r_{0} / w=7.5$. On this basis, the variation of $S_{\mathrm{e} i}$ for the probes was evaluated and the results for the inner probes $(i=1, \ldots 4)$ are illustrated in Figure 4.

In comparison to the $S_{\mathrm{e} i}$ results shown in [1] for the inner probes, $S_{\mathrm{e} i}$ does not attain a value $>1$. The reduction in the $S_{\mathrm{e} i}$ value is of course a consequence

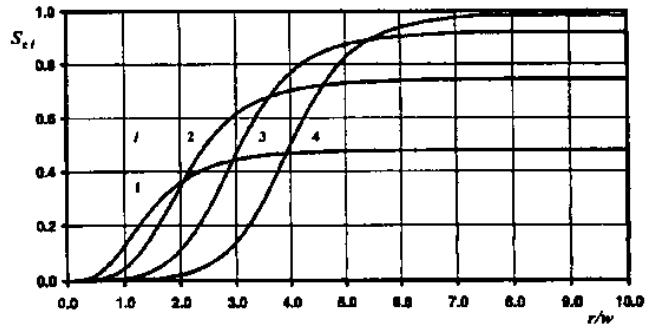

Figure 4: Variation of $S_{\mathrm{e} i}$ for the inner probes.

of the reduced $\lambda_{i}$ values, with probes 1 and 2 exhibiting the greatest reductions. The $S_{\mathrm{e} i}$ results for the outer probes $(i=5, \ldots 8)$ are essentially identical to the results discussed in [1].

For the same surface charge conditions, the charge $q_{\mathrm{p}}$ induced on the point electrode is given by

$$
q_{p}(r)=-2 \pi \sigma_{0} \int_{0}^{r} \lambda_{p} r^{\prime} \mathrm{d} r^{\prime}
$$

where $r^{\prime}$ is again a dummy variable.

Owing to the very different forms of $\lambda_{i}$ and $\lambda_{p}$, it is of interest to consider the relation between $q_{i}$ and $q_{\mathrm{p}}$. The basis for a comparison may be obtained by combining (4) and (7) to give

$$
\frac{q_{i}}{q_{\mathrm{p}}}=\frac{\int_{0}^{r} \lambda_{i} r^{\prime} \mathrm{d} r^{\prime}}{\int_{0}^{r} \lambda_{\mathrm{p}} r^{\prime} \mathrm{d} r^{\prime}}
$$

The variation of the ratio $q_{i} / q_{\mathrm{p}}$ is illustrated in Figure 5 for the inner probes.

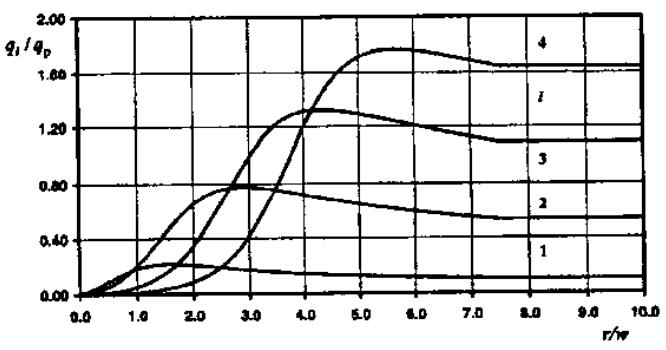

Figure 5: Variation of $q_{i} / q_{\mathrm{p}}$ for the inner probes. 
For probes 1 and 2 , it is seen that, as $q_{i} / q_{p}(1$, the charge induced on the point is greater than that induced on probe. With the exception of probe 8 , see [1], the maximum value of $q_{i} / q_{\mathrm{p}}>1$ for the remaining probes, implying that the charge induced on these probes is greater than that induced on the point electrode.

The above behaviour can be understood with respect to the different $\lambda$ functions. The values of $\lambda_{1}$ and $\lambda_{2}$ are reduced markedly owing to their proximity to the point electrode, while $\lambda_{p}$ decreases rapidly with distance from axis of the system.

\section{Discussion}

We have examined the response of a system of annular probes with reference to a planar dielectric. In the present study, the existence of the high-voltage electrode was taken into account in the evaluation of the probe $\lambda$ functions. It is shown that this electrode has a significant influence on the $\lambda$ functions of the probes nearest to it, in this instance the inner probes ( $i$ $=1, \ldots 4)$. The influence is further reflected in the response of these inner probes.

For real-time measurements, during which a high voltage is applied to the point electrode, an additional aspect arises because the surface charge produced by the discharge induces charge on all electrodes in the system.

Depending on the electrode in question, these induced charges can produce changes in the electrode potentials. Through the partial capacitances these changes are registered at the measuring probe. Such a situation will make the interpretation of the probe measurements more difficult.

The significance of this situation has been demonstrated by assessing the ratio of the induced charge on a probe $q_{i}$ to that on the point electrode $q_{\mathrm{p}}$.

It is shown that $q_{i} / q_{\mathrm{p}}<1$ for the innermost probes $(i=$ $1,2)$. This suggests that the charge induced on the point can provide a significant fraction of the signals from these probes, leading to additional difficulties in the interpretation of measurements from probes $1 \& 2$.

In [2-4], the authors made simultaneous measurements from 4 probes. This implies that the signal from any probe reflects not only the induced charge from the point electrode, but also from the 3 other probes. Hence, without a knowledge of the relevant partial capacitances and changes in potential, a quantitative analysis of a probe record is not feasible.

\section{Conchusion}

For electrostatic probe measurements undertaken during the voltage application, the existence of the high-voltage electrode must be considered in the evaluation/analysis of the probe signals. This requirement arises because the electrode influences the $\lambda$ function of the nearest probes, and thus the response of these probes. In addition, the charge induced on the high-voltage electrode is registered, via the partial capacitance and change in potential, at the measuring probe.

\section{Acknowledgment}

The authors wish to thank J. Berril for producing the WORD document.

\section{References}

[I] T. Johansson and I. W. McAllister, "Response of an annular electrostatic probe for a planar dielectric spacer", 2003 Annual Report - Conference on Electrical Insulation and Dielectric Phenomena, Albuquerque, pp. 469-472, 2003.

[2] O. Yamamoto, T. Hara and M. Hayashi, "Space charge distribution of surface discharge in $\mathrm{SF}_{6}$ ", Fifth International Symposium on High Voltage Engineering, Braunschweig, paper 15.12, 1987.

[3] O. Yamamoto, T. Hara, M. Matsumura and M. Hayashi, "Properties of space charge due to corona on an insulator surface in $\mathrm{SF}_{6}$ ", IX International Conference on Gas Discharges and their Applications, Venezia, pp.231-234, 1988.

[4] O. Yamamoto, T. Hara and M. Hayashi, "Measurement of streamer charge distribution on an insulator in $\mathrm{SF}_{6}$ gas", Electrical Engineering in Japan, vol.111, no.3, pp.47-56, 1991.

[5] M. Yashima, H. Fujinami and T. Takuma, "Measurement of accumulated charge on dielectric surfaces with an electrostatic probe", in L. G. Christophorou and D. W. Bouldin (eds.), Gaseous Dielectrics V. Pergamon New York, pp.242-247, 1987.

[6] T. O. Rerup, G. C. Crichton and I. W. McAllister, "Using the $\lambda$ function to evaluate probe measurements of charged dielectric surfaces", IEEE Trans. Diel. Elect. Insul., vol.3, pp. $770-777,1996$.

[7] T. Takuma, M. Yashima and T. Kawamoto, "Principle of surface charge measurement for thick insulating specimens", IEEE Trans. Diel. Elect. Insul., vol.5, pp.497-504, 1998.

[8] A. Pedersen, "On the electrostatics of probe measurements of surface charge densities", in L G. Christophorou and D. W. Bouldin (eds.), Gaseous Dielectrics V, Pergamon New York, pp.235-240, 1987. 